\title{
Retraction
}

\section{Retracted: Bifurcation of Travelling Wave Solutions of the Generalized Zakharov Equation}

\author{
Journal of Applied Mathematics \\ Received 27 April 2016; Accepted 27 April 2016 \\ Copyright (C) 2016 Journal of Applied Mathematics. This is an open access article distributed under the Creative Commons \\ Attribution License, which permits unrestricted use, distribution, and reproduction in any medium, provided the original work is \\ properly cited.
}

Journal of Applied Mathematics has retracted the article titled "Bifurcation of Travelling Wave Solutions of the Generalized Zakharov Equation" [1]. The article was previously published as "Classification of bounded travelling wave solutions of the generalized Zakharov equation,” by H. R. Z. Zangeneh, R. Kazemi and M. Mosaddeghi, in Iranian Journal of Science \& Technology.

\section{References}

[1] M. Mosaddeghi, "Bifurcation of travelling wave solutions of the generalized Zakharov equation," Journal of Applied Mathematics, vol. 2014, Article ID 170946, 11 pages, 2014. 


\title{
Bifurcation of Travelling Wave Solutions of the Generalized Zakharov Equation
}

\author{
Masoud Mosaddeghi \\ Département de Mathématiques et de Statistique, Université de Montréal, CP 6128, Succursale Centre-Ville, \\ Montréal, QC, Canada H3C 3J7
}

Correspondence should be addressed to Masoud Mosaddeghi; mosaddeghi@dms.umontreal.ca

Received 11 April 2014; Accepted 26 July 2014; Published 24 August 2014

Academic Editor: Tiecheng Xia

Copyright (C) 2014 Masoud Mosaddeghi. This is an open access article distributed under the Creative Commons Attribution License, which permits unrestricted use, distribution, and reproduction in any medium, provided the original work is properly cited.

By using bifurcation theory of planar ordinary differential equations all different bounded travelling wave solutions of the generalized Zakharov equation are classified in to different parametric regions. In each of these parametric regions the exact explicit parametric representation of all solitary, kink (antikink), and periodic wave solutions as well as their numerical simulation and their corresponding phase portraits are obtained.

\section{Introduction}

Many phenomena in physics, engineering, and science are described by nonlinear partial differential equations (NPDEs). Exact travelling wave solution of nonlinear evolution equation is one of the fundamental objects of study in mathematical physic. When these exact solutions exist, they can help one to understand the mechanism of the complicated physical phenomena and dynamical processes modelled by these nonlinear evolution equations. In the past decades a vast variety of the powerful and direct methods to find the explicit solutions of NPDE have been developed, such as Hirota bilinear method $[1,2]$, inverse scattering transform method [3], Backlund and Darboux transforms method [4], Lie group method [5] F-expansion method [6], sine-cosine method [7], homotopy perurbation method [8], homogenous balance method [9], algebraic method [10], and Jacobi elliptic function expansion method [11]. Certainly, the bifurcation theory of planar dynamical systems is an efficient method too $[12,13]$. In this paper we consider the generalized Zakharov equation by using the bifurcation theory. The Zakharov equations [14] are used extensively in considerations of the evolution of Langmuir turbulence when strong turbulence effects are considered. The Zakharov equation has various applications in physics in a theory of deep-water waves [15], communication [16], and nonlinear pulse propagation in fibers [17]. The original derivation of these equations was based on a simplified model involving fluid concepts. The model leads to two equations: one of these describes the evolution of the envelope of the Langmuir waves with the nonlinearity included through a term involving a density fluctuation, and the other describes the evolution of the density fluctuation due to the ponderomotive force exerted by the Langmuir waves. Now we consider the generalized Zakharov equations which have the forms

$$
\begin{gathered}
u_{t t}-c_{s}^{2} u_{x x}=\beta\left(|E|^{2}\right)_{x x} \\
i E_{t}+\alpha E_{x x}-a_{1} u E+a_{2}|E|^{2} E+a_{3}|E|^{4} E=0 .
\end{gathered}
$$

In (1) $\alpha$ represents the coefficient of dispersion and $a_{1}, a_{2}, a_{3}$ represent the coefficients of nonlinearity. When $a_{2}=a_{3}=0$, (1) reduce to the famous Zakharov equations (see $[18,19]$ for details) which describe the propagation of Langmuir waves in plasmas. The complex number $E$ represents the envelope of the electric field, and $u$ is equilibrium value from the fluctuation of the ion density. The parameter $c_{s}$ is proportional to the ion acoustic speed. We find exact solutions of (1) via the bifurcation theory of planar dynamical systems. The purpose of this paper is to give the bifurcation sets of the bounded travelling wave solutions, that is, solitary wave solutions, kink (anti kink) wave solutions, and periodic wave solutions. Also we obtain the explicit representation for some of these solutions in different parametric region determined by the 
bifurcation set. To find the travelling wave solutions of (1) we consider the travelling wave solutions of the form

$$
\begin{array}{r}
E(x, t)=v(\xi) \exp [i(k x-\omega t)], \quad u(x, t)=u(\xi), \\
\xi=x-c t,
\end{array}
$$

where $k, \omega$, and $c$ are arbitrary constants; $c$ denotes the wave speed, $k$ is the frequency, $\omega$ is the soliton wave number, and $v(\xi)$ is a real function that represents the shape of the pulse. By substituting for $E$ and $u$ from (2) into (1) we get the following ordinary differential equations:

$$
\begin{gathered}
\left(c^{2}-c_{s}^{2}\right) u_{\xi \xi}=\beta\left(v^{2}\right)_{\xi \xi} \\
\alpha v_{\xi \xi}+i(2 \alpha k-c) v_{\xi}+\left(\omega-\alpha k^{2}\right) v-a_{1} u v+a_{2} v^{3}+a_{3} v^{5}=0 .
\end{gathered}
$$

By integration of the first equation in (3) twice with respect to $\xi$ and taking the integration constant to be zero, we obtain

$$
u=\frac{\beta}{\left(c^{2}-c_{s}^{2}\right)} v^{2}
$$

now we substitute (4) in the second equation of (3) to obtain

$$
v_{\xi \xi}+a v+b v^{3}+\gamma v^{5}=0
$$

where

$$
\begin{aligned}
a=\frac{\left(\omega-\alpha k^{2}\right)}{\alpha}, & b=\frac{a_{2}}{\alpha}-\frac{\beta a_{1}}{\left(c^{2}-c_{s}^{2}\right)}, \\
\gamma=\frac{a_{3}}{\alpha}, & c=2 \alpha k .
\end{aligned}
$$

Now let $d v / d \xi=y$. Then we drive the following travelling wave system which is a planar Hamiltonian system:

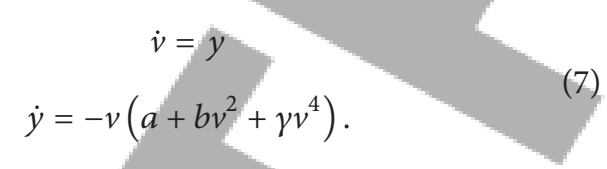

Because the phase portraits of the Hamiltonian system (7) determine travelling wave solutions of (1), we find the bifurcation set for which the qualitative behavior of phase portraits of (7) changes. Here we consider only bounded travelling waves because in physical model only bounded travelling waves are meaningful. Suppose that $v(x, t)=v(x-$ $c t)=v(\xi)$ is a continuous solution of system (7) for $-\infty<$ $\xi<\infty$ and $\lim _{\xi \rightarrow+\infty} v(\xi)=p, \lim _{\xi \rightarrow-\infty} v(\xi)=q$. We recall that

(i) if $p=q$, then $v(x, t)$ is called a solitary or impulse wave solution, and

(ii) if $p \neq q$ then $v(x, t)$ is called kink (antikink) wave solution.

Usually a solitary wave solution, a kink (antikink) wave and periodic travelling wave solutions of (1) correspond to a homoclinic orbits or cuspidal loop, heteroclinic orbit or eye-figure loop, and periodic orbit of (7), respectively. Thus we need to find all periodic orbits, homoclinic orbits, and heteroclinic orbits of system (7) which depend on the systems' parameters.

The rest of this paper is organized as follows. In Section 2, we give the bifurcation set and corresponding phase portrait of system (7). In Section 3, using the information obtained about the phase portraits of bounded solutions of (7), we obtain the numerical simulation for corresponding bounded travelling wave solutions of system (1). In Section 4, we give exact explicit parametric representation of different possible solitary wave solutions, periodic travelling wave solutions, and kink (antikink) wave solutions of (1).

\section{Bifurcation of Phase Portrait of (7)}

In this section, we consider bifurcation set and phase portraits of (7). First we consider the generic case $\gamma \neq 0$. With some time scaling and without loss of generality, we can assume $\gamma=$ \pm 1 , so that we can have Hamiltonian system:

$$
\begin{gathered}
\dot{v}=y, \\
\dot{y}=-v\left(a+b v^{2} \pm v^{4}\right):=f_{ \pm}(v),
\end{gathered}
$$

with the Hamiltonian $H_{ \pm}(v, y)=y^{2} / 2+F_{ \pm}(v)$, where \pm correspond to $\gamma= \pm 1$ and $F_{ \pm}(v)= \pm v^{6} / 6+b v^{4} / 4+a v^{2} / 2$ is the corresponding potential function. It is clear that critical points of $F_{ \pm}$are zeros of $f_{ \pm}$. It is known that isolated minimum, maximum, and inflection points of $F$ correspond to center, saddle point, and cusp point of system (8), respectively (e.g., see [20]). Also it is known that the global structure of phase portraits of system (8) will not change qualitatively unless one of the conditions listed below is violated [20].

(i) There are only finitely many critical points of $F_{ \pm}$.

(ii) Each critical point of $F_{ \pm}$is nondegenerate; that is, $F_{ \pm}^{\prime \prime}\left(\bar{v}_{1}\right) \neq 0$ for critical point $\bar{v}_{1}$.

(iii) No two maximum values of $F_{ \pm}$are equal.

(iv) $\left|F_{ \pm}\left(v_{1}\right)\right| \rightarrow \infty$ as $\left|v_{1}\right| \rightarrow \infty$; that is, $F_{ \pm}$is unbounded for both $v_{1} \rightarrow \infty$ and $v_{1} \rightarrow-\infty$.

Potential functions satisfying the above four conditions are called the generic potential functions. In our case it is clear that conditions $i$ and $i v$ are satisfied for all values of $a$ and $b$. Therefore to find the bifurcation set, we first need to find conditions where critical points of $F_{ \pm}$become degenerate. So we set

$$
\begin{gathered}
f_{ \pm}(a, b, v)=a v+b v^{3} \pm v^{5}=0, \\
\frac{\partial f_{ \pm}(a, b, v)}{\partial v}=a+3 b v^{2} \pm 5 v^{4}=0 .
\end{gathered}
$$

By solving (9) we find the bifurcation set to be $B_{+}=\{(a, b)$ : $\left.a=0, b^{2}=4 a, b<0\right\}$ and $B_{-}=\left\{(a, b): a=0, b^{2}=-4 a\right.$, $b>0\}$ which correspond to $\gamma= \pm 1$, respectively. These bifurcation sets divide the parametric plane into 7 distinct regions (see Figures 1 and 2). In each parametric region, the number and type of critical points remain unchanged. To 


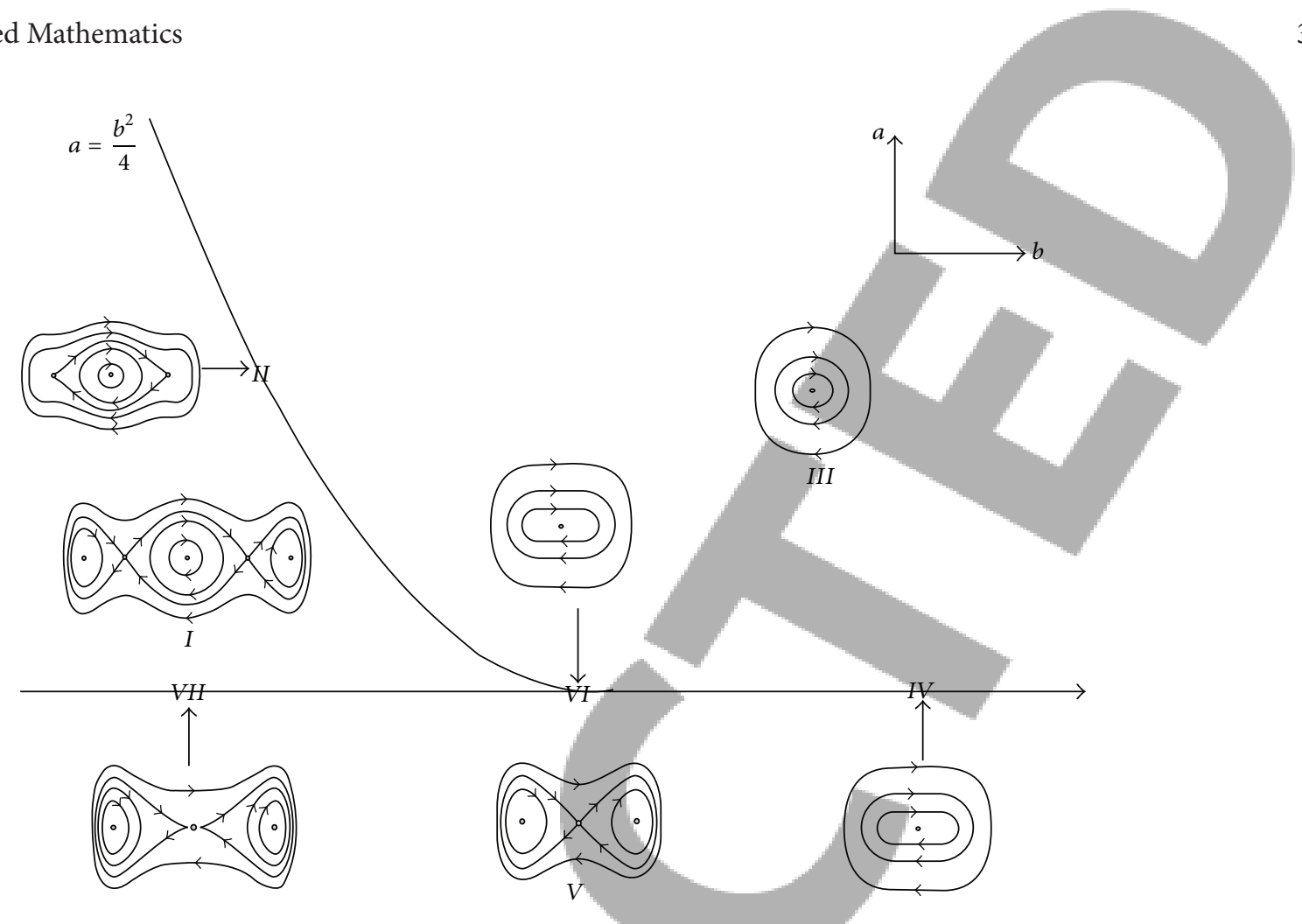

FIGURE 1: Bifurcation sets and phase portraits of (8) with $\gamma=1$ in different parametric regions.

see the type and number of critical points it is sufficient to consider only a typical equation for a particular value of $a$ and $b$ in each region. Critical points of $F_{ \pm}$are $v=0$ and $v=$ $\pm \sqrt{\left(-b \pm \sqrt{\Delta_{ \pm}}\right) /( \pm 2)}$ where $\Delta_{ \pm}=b^{2} \mp 4 a$ which correspond to $\gamma= \pm 1$, respectively. It is easy to verify that number of critical points of $F_{ \pm}$will change from one nondegenerate critical point in region III to five nondegenerate critical points in region $(I)$ and three nondegenerate critical points in region $(V)$. On the boundary of these regions, that is, regions $I I, I V, V I$, and $V I I$, critical points are degenerate. To classify the critical points and determine the phase portraits of system (7) we need to consider the two cases $\gamma=1$ and $\gamma=-1$ separately.

Case $I(\gamma=1)$. Since $F$ is a sixth order polynomial and the coefficient of $v^{6}$ is positive, it is easy to verify that the only nondegenerate critical point of $F$ in region III $\left(\Delta_{+}<0\right.$ or $\left.\Delta_{+} \geq 0, b>0, a>0\right)$ is $v=0$ which is a minimum point. Therefore in this region system (7) has a global center (see Figure 1).

In region II $\left(\Delta^{+}=0, b<0\right), F_{+}$has three critical points where $v=0$ is a minimum and $v= \pm \sqrt{-b / 2}$ are inflection points. These points correspond to a center and cusp points of (7), respectively. Also because of symmetry, $H_{+}(\sqrt{-b / 2}, 0)=$ $H_{+}(-\sqrt{-b / 2}, 0)>H(0,0)$. Therefore system (7) will have an eye-figure loop connecting the cusp points and an oval of periodic orbits encircling the origin and also a band of periodic orbits outside the eye-figure loop (see Figure 1).

In region $I\left(\Delta_{+}>0, b<0, a>0\right)$ the potential function $F_{+}$always has three nondegenerate minimum points and two nondegenerate maximum points between them. Therefore in this region system (7) has three center and two saddle points. Furthermore, since $F_{+}$is even and the critical points $\pm \sqrt{\left(-b-\sqrt{\Delta_{+}}\right) / 2}$ are symmetric with respect to $a$-axis, we have

$$
F_{+}\left(\sqrt{\frac{\left(-b-\sqrt{\Delta_{+}}\right)}{2}}, 0\right)=H_{+}\left(-\sqrt{\frac{\left(-b-\sqrt{\Delta_{+}}\right)}{2}}, 0\right) .
$$

There will be a cycle, heteroclinic to these two saddle points, and since

$$
H_{+}(0,0)<H_{+}\left( \pm \sqrt{\frac{\left(-b-\sqrt{\Delta_{+}}\right)}{2}}, 0\right),
$$

this heteroclinc cycle includes a band of periodic orbits encircling the origin. Also since $H_{+}\left(-\sqrt{\left(-b-\sqrt{\Delta_{+}}\right) / 2}, 0\right)>$ $H_{+}\left(-\sqrt{\left(-b+\sqrt{\Delta_{+}}\right) / 2}, 0\right)$ and $H_{+}\left(\sqrt{\left(-b-\sqrt{\Delta_{+}}\right) / 2}, 0\right)>$ $H_{+}\left(\sqrt{\left(-b+\sqrt{\Delta_{+}}\right) / 2}, 0\right)$, there will be two orbits homoclinic to these saddle points. These homoclinc orbits include a band of periodic orbits encircling centers at $\pm \sqrt{\left(-b+\sqrt{\Delta_{+}}\right) / 2}$ points of system (7) (see Figure 1).

In regions $I V(b>0, a=0)$ and $V I, a=b=0$ the only critical point of $F_{+}$is $v=0$ which is a degenerate minimum point; therefore system (7) has a global degenerate center at origin (see Figure 1).

In region $V(a<0), F_{+}$has three nondegenerate critical points at $v=0$ and $v= \pm \sqrt{\left(-b+\sqrt{\Delta_{+}}\right) / 2}$, where $v=0$ is 

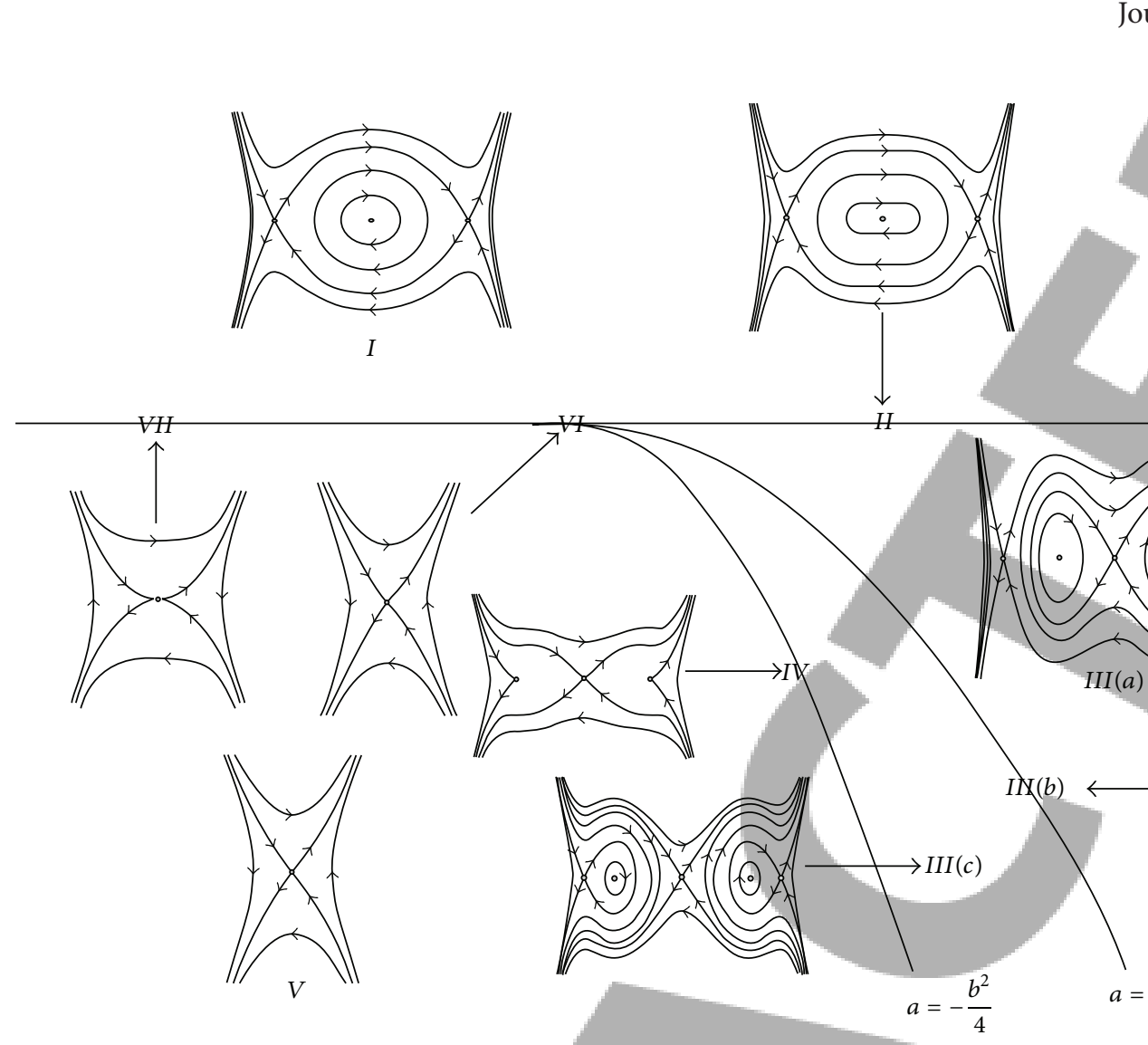

$$
a=-\frac{b^{2}}{4} \quad a=-\frac{3}{16} b^{2}
$$

FIGURE 2: Bifurcation sets and phase portraits of (8) with $\gamma=-1$ in different parametric regions.

a maximum and $v= \pm \sqrt{\left(-b+\sqrt{\Delta_{+}}\right) / 2}$ are minimum points. Also $H_{+}(0,0)>H\left( \pm \sqrt{\left(-b+\sqrt{\Delta_{+}}\right) / 2}, 0\right)$. Therefore system (7) has orbits double homoclinc to saddle point at the origin. Also there will be ovals of periodic orbits inside each of the homoclinic orbits encircling centers at $\left( \pm \sqrt{\left(-b+\sqrt{\Delta_{+}}\right) / 2}, 0\right)$ and a band of periodic orbits outside the double homoclinic (figure-eight loop) orbits (see Figure 1).

In region VII $(a=0, b<0)$, the situation is similar to that of region $V$ with the difference that the maximum point of $F_{+}$ and saddle point of system (7) are degenerate in this case (see Figure 1).

Therefore we have proved the following lemma.

Lemma 1. Phase portrait of system (7) corresponding to $H_{+}=$ $h$ is classified as follows (see Figure 1):

(i) In region $I\left(\Delta_{+}>0, a>0, b<0\right)$, phase portrait of system (8) consists of two saddle points, three centers, $a$ cycle heteroclinic to saddle points, two homoclinic orbits to saddle points, and bands of periodic orbits inside heteroclinic cycle and homoclinic orbits and outside figure-eight loop.

(ii) In region (II) $\left(\Delta_{+}=0, b<0\right)$ phase portrait of (7) consists of a center, two cusp points, an eye-figure loop, and two bands of periodic orbits inside and outside the eye-figure loop. (iii) In regions III $\left(\Delta_{+}<0\right.$ or $\left.\Delta_{+} \geq 0, a>0, b>0\right)$ phase portrait consists of a global nondegenerate center.

(iv) In regions $V I(a=b=0)$ and $I V(a=0, b>0)$, phase portrait consists of a degenerate global center.

(v) In region $V(a<0)$, phase portrait consists of $a$ nondegenerate saddle point at the origin, two centers at $\left( \pm \sqrt{\left(-b+\sqrt{\Delta_{+}}\right) / 2}, 0\right)$, a double homoclinic orbit to the saddle point, and bands of periodic orbits inside and outside the double homoclinic orbit.

(vi) In region VII $(a=0, b<0)$, phase portrait consists of a degenerate saddle point at the origin, two centers, a double homoclinic orbit to the saddle point, and bands of periodic orbits inside and outside the double homoclinic orbit.

Case II $(\gamma=-1)$. In this case parametric region is divided into eight locally topologically equivalent regions by bifurcation set $B_{-}$(see Figure 2 ). Using a similar analysis to Case I, we can derive the phase portraits of system (7). In region $I I I\left(\Delta_{-}>0\right.$, $a<0, b>0)$ potential function $F_{-}$has three nondegenerate maximum points at $x=0, x= \pm \sqrt{\left(b+\sqrt{\Delta_{-}}\right) / 2}$ and two minimum points at $x= \pm \sqrt{\left(b-\sqrt{\Delta_{-}}\right) / 2}$. Also because of symmetry, $H_{-}\left(\sqrt{\left(b+\sqrt{\Delta_{-}}\right) / 2}, 0\right)=H_{-}\left(-\sqrt{\left(b+\sqrt{\Delta_{-}}\right) / 2}, 0\right)$. Therefore saddle points $\pm\left(\sqrt{\left(b+\sqrt{\Delta_{-}}\right) / 2}, 0\right)$ always lie on 
the same level curve of the Hamiltonian $H_{-}=h$. Furthermore along parabola $a=-3 b^{2} / 16, b>0$, all three saddle points lie on the same potential level; that is, $H(0,0)=$ $H\left(\sqrt{\left(b+\sqrt{\Delta_{-}}\right) / 2}, 0\right)=0$; therefore region III will be divided into three subregions which we will denote by $\operatorname{III}(a)=$ $\left\{(a, b) \in\right.$ region III $\left.: a>-3 b^{2} / 16\right\}, \operatorname{III}(b)=\left\{(a, b): a=-3 b^{2}\right.$ $/ 16\}$, and $\operatorname{III}(c)=\left\{(a, b): a<-3 b^{2} / 16\right\}$. In region III $(b)$ bounded orbits of phase portraits of system (7) consist of two cycles heteroclinic to saddle points at origin and $\left(\sqrt{\left(b+\sqrt{\Delta_{-}}\right) / 2}, 0\right)$ and bands of periodic orbit inside the heteroclinic cycles. In region $\operatorname{III}(a), 0=H(0,0)<$ $H_{-}\left( \pm \sqrt{\left(b+\sqrt{\Delta_{-}}\right) / 2}, 0\right)$; therefore the phase portrait of bounded orbits of (7) consists of a double homoclinic orbit to origin, a cycle heteroclinic to saddle points $\left( \pm \sqrt{\left(b+\sqrt{\Delta_{-}}\right) / 2}, 0\right)$, bands of periodic orbits inside homoclinic orbits, and a band of periodic orbits outside double homoclinic orbit and inside the heteroclinic cycles. In region $\operatorname{III}(c), 0=H(0,0)>H_{-}\left( \pm \sqrt{\left(b+\sqrt{\Delta_{-}}\right) / 2}, 0\right)$. Therefore the phase portraits of bounded periodic orbits consists of homoclinic orbits to saddle points $\left( \pm \sqrt{\left(b+\sqrt{\Delta_{-}}\right) / 2}, 0\right)$ and band of periodic orbits inside each of the homoclinic orbits (see Figure 2). Phase portraits of system (7) in other regions were derived in a similar manner, omitted here for the sake of brevity. Therefore we proved the following.

Lemma 2. Phase portrait of system (7) corresponding to $H_{-}$ $h$ is classified as follows (see Figure 2).

(i) In region $I(a>0)$ phase portrait of bounded orbits consists of a center at the origin, two saddle points at $\left( \pm \sqrt{\left(b+\sqrt{\Delta_{-}}\right) / 2}, 0\right)$, cycle heteroclinic to the saddle points, and a band of periodic orbits encircling the origin inside the heteroclinic cycle.

(ii) In region II ( $a=0, b>0$ ), phase portrait of bounded orbits consists of a degenerate center at the origin, two saddle points at $( \pm \sqrt{b}, 0)$, cycle heteroclinic to the saddle points, and a band of periodic orbits encircling the origin inside the heteroclinic cycle.

(iii) In region (III) $\left(\Delta_{-}>0, a<0, b>0\right)$, it has three saddle points at $(0,0),\left( \pm \sqrt{\left(b+\sqrt{\Delta_{-}}\right) / 2}, 0\right)$, and two centers at $\left( \pm \sqrt{\left(b-\sqrt{\Delta_{-}}\right) / 2}, 0\right)$. In regions $\operatorname{III}(a)$ there are a double homoclinic orbit to origin, a cycle heteroclinic to saddle points $\left(\sqrt{\left(b+\sqrt{\Delta_{-}}\right) / 2}, 0\right)$, bands of periodic orbits inside homoclinic orbits, and a band of periodic orbits outside double homoclinic orbit and inside the heteroclinic cycles. In region III (b) there are two cycles heteroclinic to saddle points at origin and $\left(\sqrt{\left(b+\sqrt{\Delta_{-}}\right) / 2}, 0\right)$ and bands of periodic orbit inside the heteroclinic cycles. In region $\operatorname{III}(c)$ there are homoclinic orbits to saddle points $\left( \pm \sqrt{\left(b+\sqrt{\Delta_{-}}\right) / 2}, 0\right)$ and band of periodic orbits inside each of the homoclinic orbits. (iv) In region IV $\left(\Delta_{-}=0, b>0, a<0\right)$, there are a saddle point at $(0,0)$ and two cusp points at $( \pm \sqrt{b / 2}, 0)$.

(v) In region $V\left(\Delta_{-}<0\right.$ or $\left.\Delta_{-} \geq 0, a<0, b<0\right)$ there is only a saddle point at $(0,0)$.

(vi) In regions VII $(a=0, b<0)$ and VI $(a=b=0)$, there is a nonhyperbolic saddle point at the origin $(0,0)$.

Case III $(\gamma=0)$. Now we consider the degenerate case $\gamma=0$. If $b \neq 0$, (7) becomes

$$
\begin{gathered}
\dot{v}=y, \\
\dot{y}=-v\left(a+b v^{2}\right) .
\end{gathered}
$$

Then without loss of generality we can assume $b= \pm 1$, so that we can have the Hamiltonian system

$$
\begin{gathered}
\dot{v}=y, \\
\dot{y}=-v\left(a \pm v^{2}\right):=\widetilde{f}_{ \pm}(v),
\end{gathered}
$$

with Hamiltonian $\widetilde{H}_{ \pm}(v, y)=y^{2} / 2+\widetilde{F}_{ \pm}(v)$, where \pm correspond to $b= \pm 1$ and $\widetilde{F}_{ \pm}(v)= \pm v^{4} / 4+a v^{2} / 2$ is the corresponding potential function. Similar to the above discussions of the Cases I and II, we see that bifurcation occurs in $a=0$. If $\gamma=b=0$, then system (5) becomes a linear differential equation. Then we have the following lemma.

Lemma 3. Phase portrait of system (12) corresponding to $\widetilde{H}_{ \pm}=$ $h$ is classified as follows.

(i) If $b=1$, then the phase portrait of system (12) consists of a global center at the origin for $a>0, a$ degenerate global center at the origin for $a=0$ and a nondegenerate saddle point at the origin, two centers at $( \pm \sqrt{-a}, 0)$, a double homoclinic orbit to the saddle point, and bands of periodic orbits inside and outside the double homoclinic orbit for $a<0$.

(ii) If $b=-1$, then the phase portrait of system (12) consists of two nondegenerate saddle points at $( \pm \sqrt{a}, 0)$, cycle heteroclinic to the saddle points, and a band of periodic orbits encircling the origin inside the heteroclinic cycle for $a>0$, a degenerate saddle at the origin for $a=0$, and $a$ saddle at the origin for $a<0$.

(iii) If $b=0$, then for $a=1$ we have a global center at the origin and for $a=-1$ we have a nondegenerate saddle point at the origin.

\section{The Numerical Simulation of Bounded Travelling Waves}

It is well known that the bounded travelling waves $E(\xi)$ of system (1) correspond to the bounded integral curves of system (7). In Lemmas 1, 2, and 3, we have classified all bounded integral curves of system (7). In this section we give numerical simulation for a typical member of bounded 


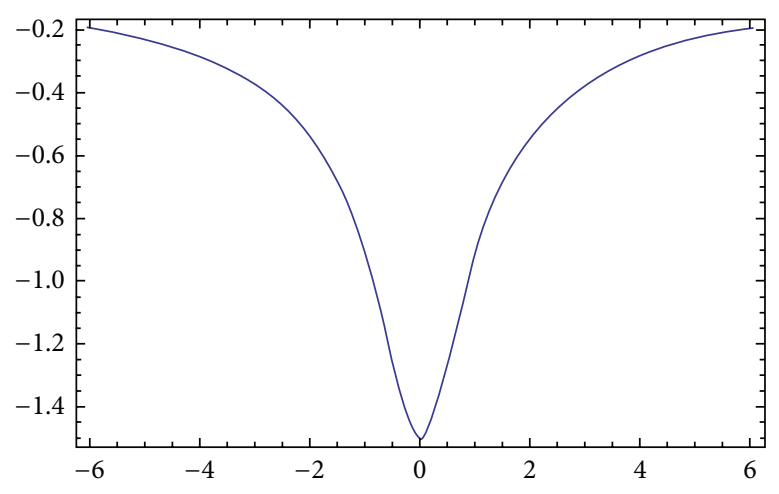

(a)

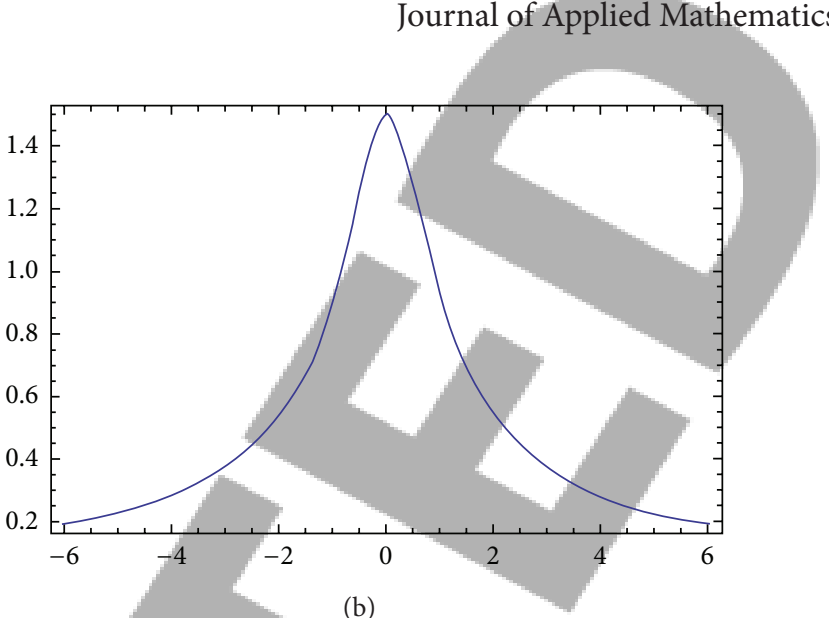

(b)

FIGURE 3: The simulation of solitary waves corresponding to the homoclinic orbits of (7). (a) Solitary wave of valley form. (b) Solitary wave of peak type.

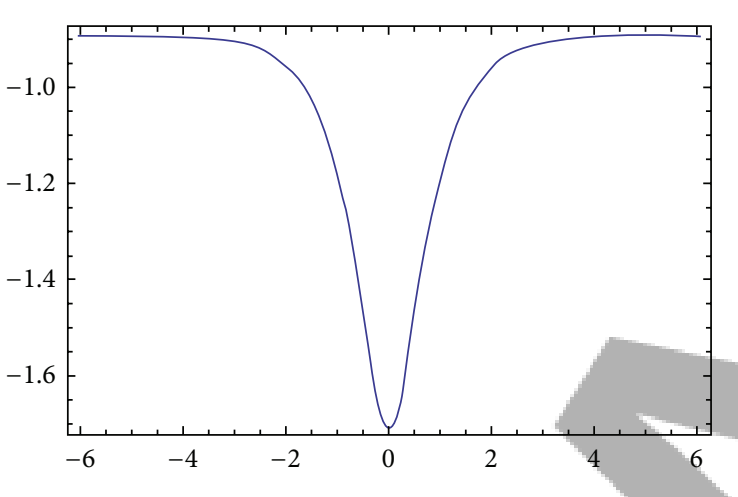

(a)

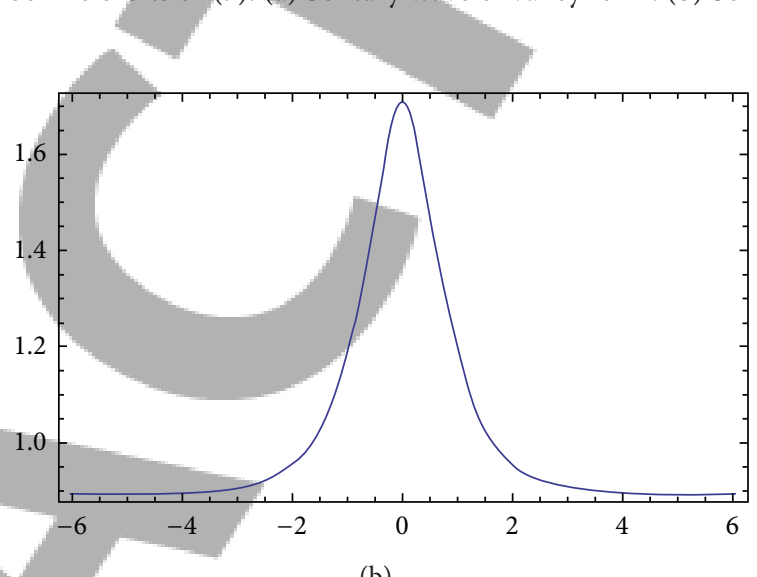

(b)

FIGURE 4: The simulation of the solitary waves corresponding to nilpotent homoclinic orbits of (7). (a) Solitary wave of valley form. (b) Solitary wave of peak form.

travelling waves of system (7) in the form of $v(x, t)=v(x-$ $c t)=v(\xi)$ as follows.

Case I (homoclinic loops). These orbits only exist in regions $I, V$ when $\gamma=1$ and in regions $\operatorname{III}(a), \operatorname{III}(c)$ when $\gamma=-1$ and for $\gamma=0, a<0$. Homoclinic orbits of system (7) correspond to solitary travelling waves of (5). Let $\gamma=1$, $b=-3$, and $a=7 / 4$ which correspond to a point in region $I$ of Figure 1. Now we consider system (7) and choose initial conditions $v(0)=-1.707106, v^{\prime}(0)=0$. So, they lie on the left branch of double homoclinic orbit (figure-eight loop). In physics this type of travelling waves is called solitary wave with valley form (see Figure 3(a)). Now let $\gamma=1, b=-3$, and $a=7 / 4$ which correspond to a point in parametric region $I$ in Figure 1. Again we use initial conditions to be on the homoclinic orbit of system (7). Let $v(0)=1.707106, v^{\prime}(0)=0$, so that they lie on the right branch of double homoclinic orbit (figure-eight loop). This type of travelling wave in physics is called solitary wave with peak form (see Figure 3(b)).

Case II (nilpotent homoclinic loops). These orbits only exist in region VII when $\gamma=1$. Nilpotent Homoclinic loop of system (7) corresponds to solitary wave of system (5). As in the previous part we choose two sets of parameters $\gamma=1$, $b=-3 / 2$, and $a=0$ which correspond to a point in region VII in Figure 1. Now we consider system (7) and choose initial conditions $v(0)=-1.066517046, v^{\prime}(0)=0$, so that they lie on the left branch of degenerate double homoclinic (figure-eight loop) orbits which correspond to solitary wave with valley form (see Figure 4(a)). For the same parameters and initial conditions $v(0)=1.066517046, v^{\prime}(0)=0$, so that they lie on the right branch of degenerate double homoclinic (figure eight loop) orbits which correspond to solitary wave with peak form (see Figure 4(b)).

Case III (heteroclinic orbits). These orbits exist only in region $I$ when $\gamma=1$ and in regions $I, I I$, and $I I I(b)$ when $\gamma=-1$ and for $\gamma=0, a>0$. Upper and lower heteroclinic orbits of system (7) correspond to kink and antikink travelling waves of system (5), respectively. Again we consider system (7) and choose $\gamma=1, b=-3$, and $a=7 / 4$ which correspond to a point in parametric region $I$ in Figure 1 . As we have mentioned above in this case we have orbits heteroclinic to saddle points $(-\sqrt{(3-\sqrt{2}) / 2}, 0)$ and $(\sqrt{(3-\sqrt{2}) / 2}, 0)$. Now we use initial conditions $v(0)=0, v^{\prime}(0)=0.7814744144$, and $v(0)=0, v^{\prime}(0)=-0.7814744144$, on upper and lower heteroclinic orbits, respectively, and we get Figures 5(a) and 5(b). 


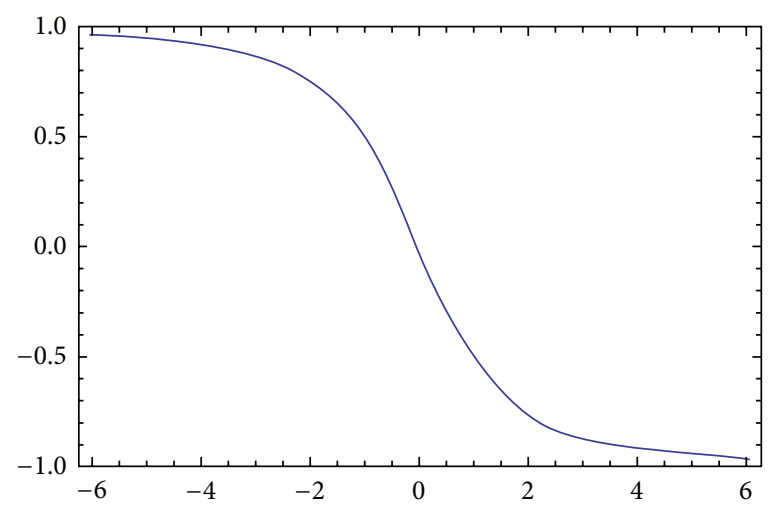

(a)

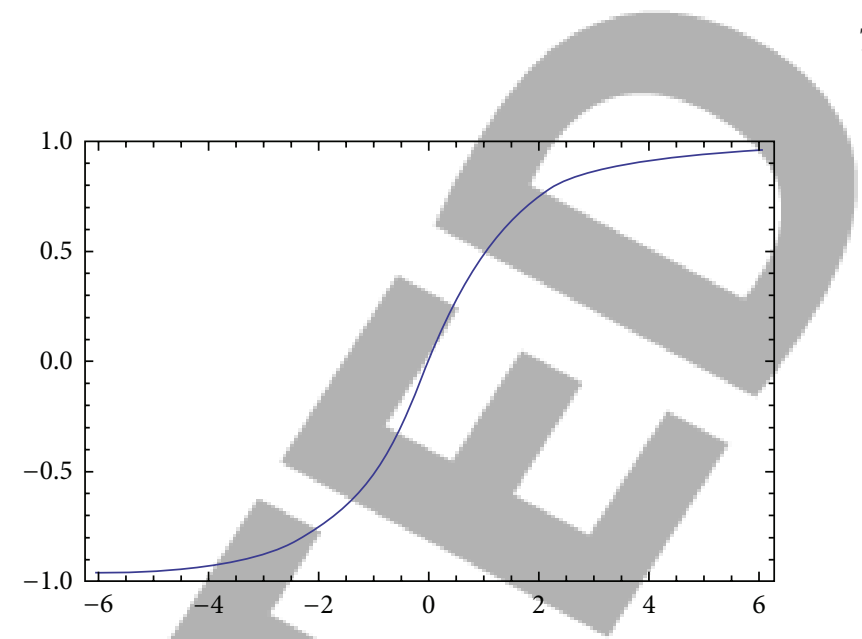

(b)

FIGURE 5: The simulation of the kink and antikink waves corresponding to the heteroclinic orbits of (7). (a)Antikink waves. (b) Kink waves.

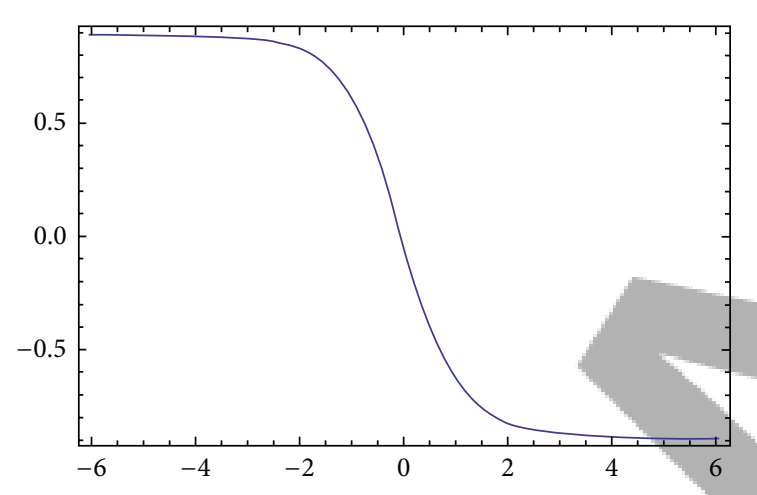

(a)

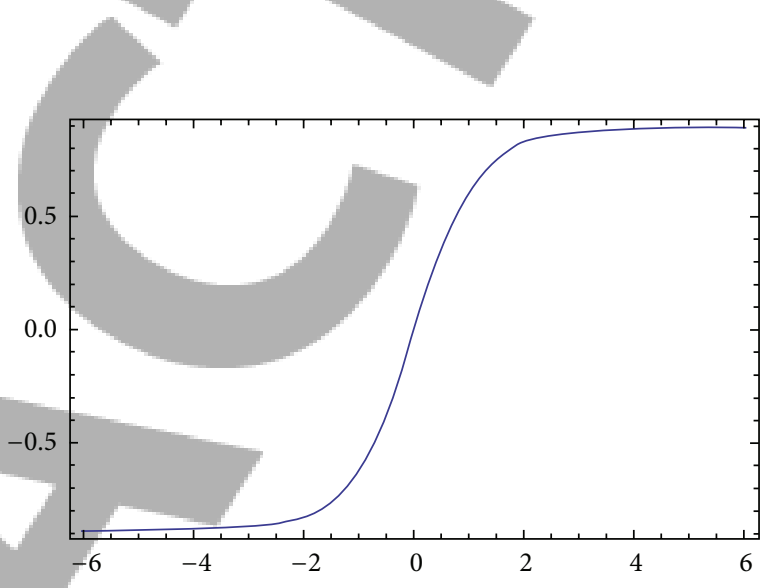

(b)

FIGURE 6: The simulation of the kink and antikink waves corresponding to the eye-figure loop of (7). (a) Antikink waves. (b) Kink waves.

Case IV (eye-figure loop). This loop exists only in region II when $\gamma=1$. Upper and lower orbits of eye-figure loop of system (7) which again correspond to kink and antikink travelling waves of system (5), respectively. As in the previous part, we choose a set of parameters $\gamma=1, b=-2$, and $a=1$ which correspond to a point in region $I I$ in Figure 1. Now we consider system (7) and choose initial conditions $v(0)=0$, $v^{\prime}(0)=0.5773502692$, and $v(0)=0, v^{\prime}(0)=-0.5773502692$, on upper and lower orbits of eye-figure loop, respectively, and we get Figures 6(a) and 6(b).

Case $V$ (periodic orbits). These periodic orbits are global (regions III, IV, VI) when $\gamma=1$ and for $\gamma=0, b=1$, $a \geq 0$ or local which lie inside homoclinic orbits (regions $I, V$ ) when $\gamma=1$ and (regions $I I I(a), I I I(c)$ ) when $\gamma=$ -1 , inside and outside of eye-figure loop (regions $I I$ ) when $\gamma=1$, inside heteroclinic cycles (region $I$ ) when $\gamma=1$ and (region $I, I I, I I I(b))$ when $\gamma=-1$ and for $\gamma=0$, $b=-1, a>0$, inside and outside of double figure-eight loop (regions $I$ ) when $\gamma=1$, inside and outside of nilpotent double homoclinic loop, (regions VII) when $\gamma=1$ and inside and outside of figure eight loop (regions $\operatorname{III}(a)$ ) when $\gamma=-1$

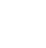



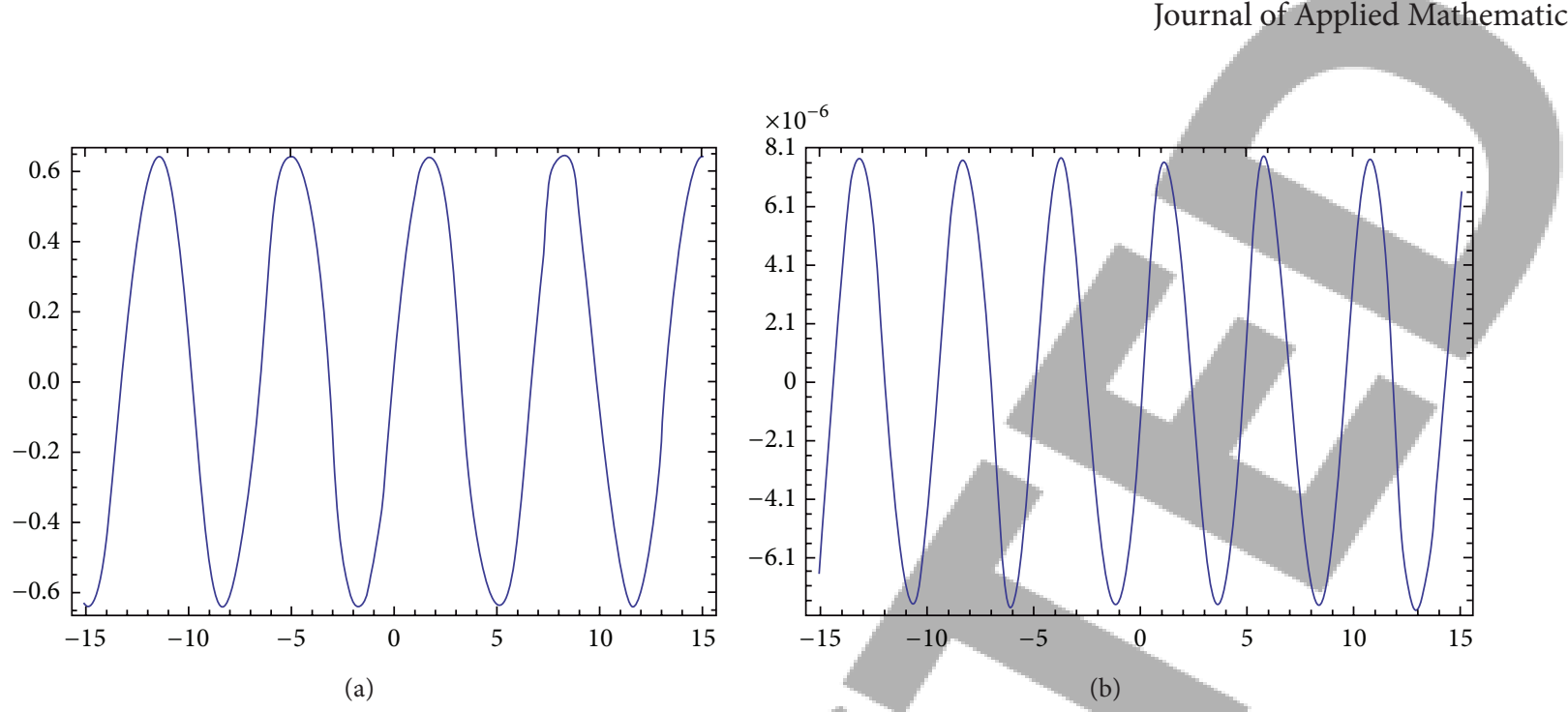

(a)

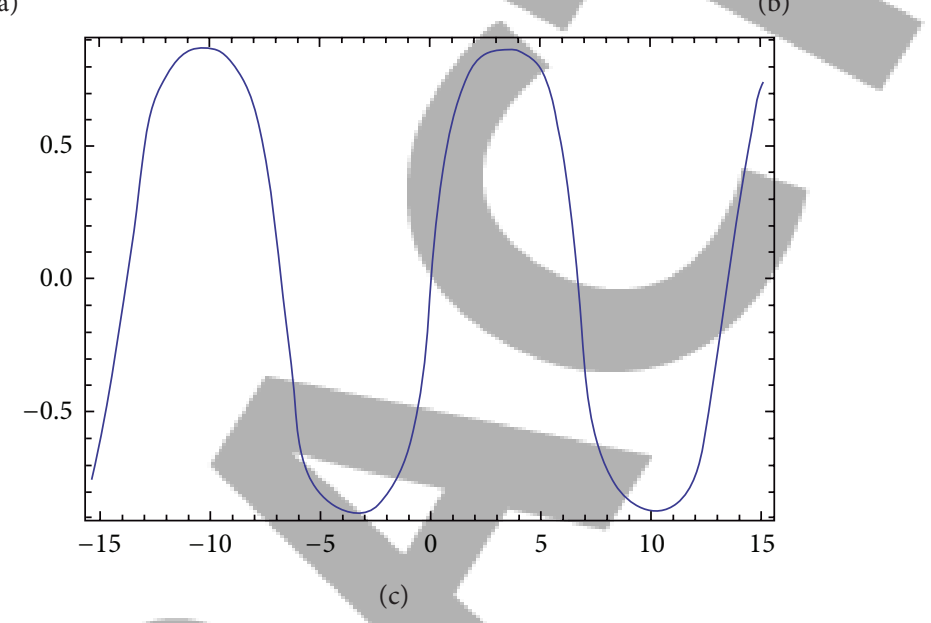

FIGURE 7: Simulation of periodic waves corresponding to periodic orbits inside heteroclinc cycle of (7). (a) Medium period. (b) Short period. (c) Long period.
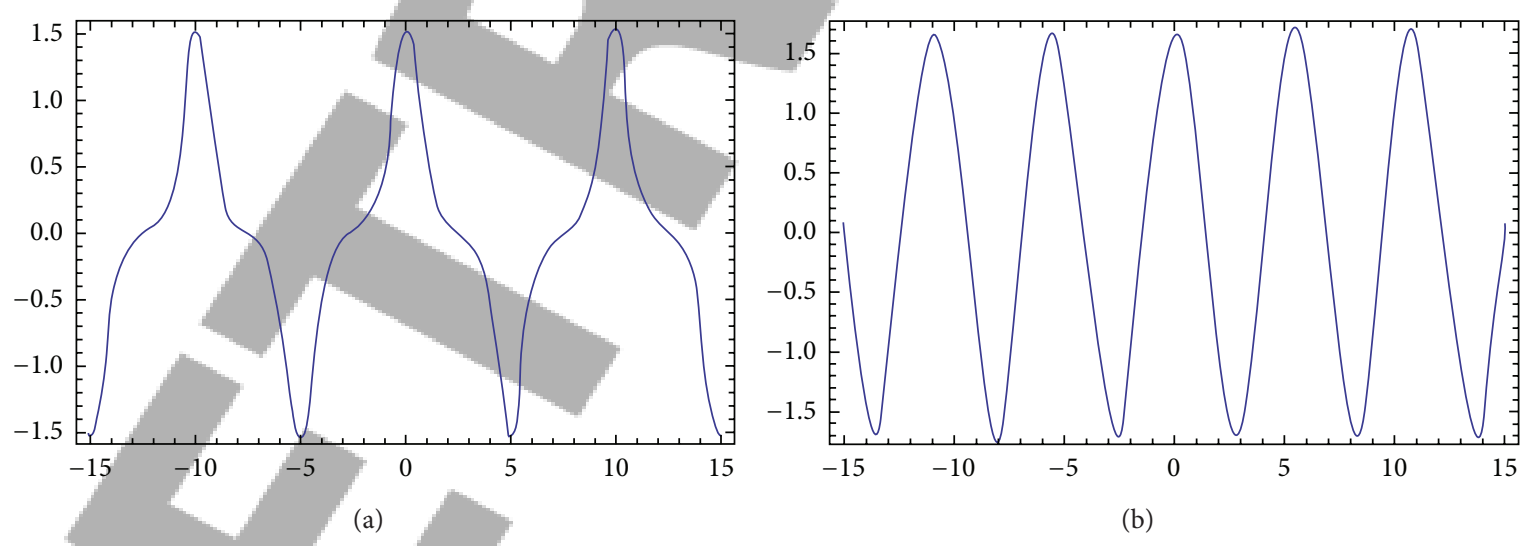

(b)

FIGURE 8: Simulation of periodic waves corresponding to periodic orbits outside figure-eight loop of (7). (a) Close to figure-eight loop. (b) Far from figure-eight loop.

close and far from the double figure-eight loop, respectively (see Figures 9(a) and 9(b)).

Remark 4. (i) We notice the difference between solitary waves in Figures 3 and 4 and kink and antikink in Figures 5 and 6 which shows their asymptotic behavior as $t \rightarrow \pm \infty$. In
Figure 3 stable and unstable manifolds of equilibrium point intersect transversally but in Figure 4 they intersect tangentially. A similar condition holds in Figures 5 and 6, respectively.

(ii) We notice that the number of inflection points on periodic waves during one-half period in each of Figures 7, 


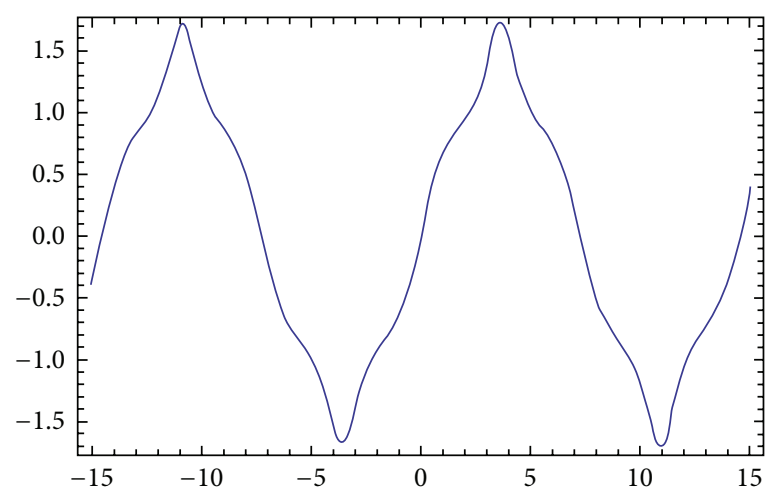

(a)

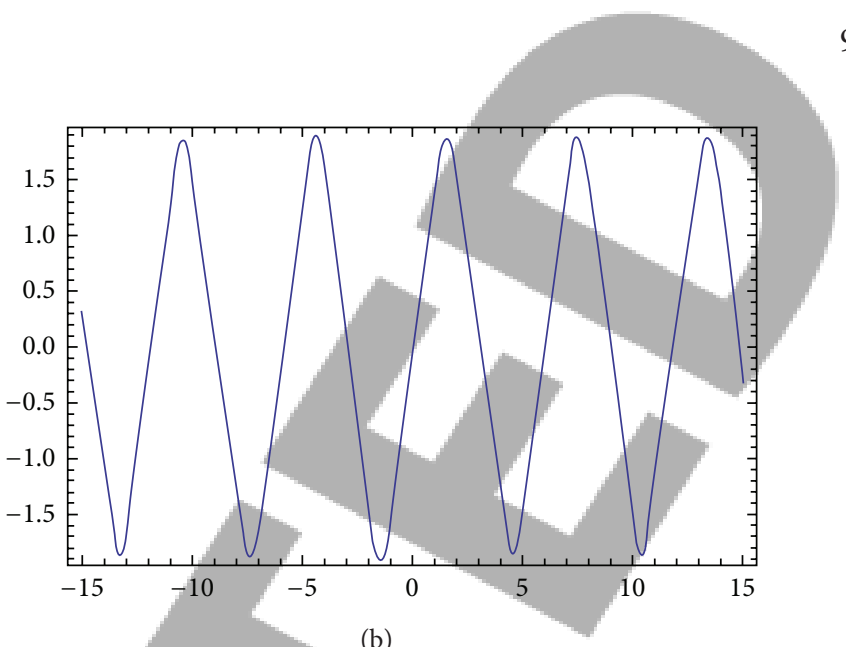

(b)

FIGURE 9: Simulation of periodic waves corresponding to periodic orbits outside double figure-eight loop of (7). (a) Close to double figureeight loop. (b) Far from double figure-eight loop.

8 , and 9 is zero, one, and two which correspond to the shape periodic orbits of system (7).

\section{Explicit Formulas for Bounded Integral Curves of (7)}

In this section we give explicit formulas for bounded travelling waves of system (7). In the first step we consider the bounded graphics of system (7).

Case $1(\gamma=+1)$

Region $\{I\}$. There are two homoclinic orbits and two heteroclinic orbits of system (7) defined by $H(v, y)=$ $H\left( \pm \sqrt{\left(-b+\sqrt{\Delta_{+}}\right) / 2}, 0\right)=H( \pm \bar{v}, 0)$ connecting saddle points $( \pm \bar{v}, 0)$ which are passing through points $( \pm \widetilde{v}, 0)$. Therefore

$$
y= \pm \sqrt{2(H(\bar{v}, 0)-H(v, 0))}= \pm\left|v^{2}-\bar{v}^{2}\right| \sqrt{\frac{\left(\widetilde{v}^{2}-v^{2}\right)}{3}}
$$

where $\widetilde{v}=\sqrt{\left(-b+2 \sqrt{\Delta_{+}}\right) / 2}$ and $\bar{v}=\sqrt{\left(-b+\sqrt{\Delta_{+}}\right) / 2}$. On the right and down branch of the homoclinic orbit we have $y=\left(v^{2}-\bar{v}^{2}\right) \sqrt{\left(\widetilde{v}^{2}-v^{2}\right) / 3}$. Since $d v / d \xi=y$, by integration along the right homoclinic orbit for negative $y$, we get:

$$
\int_{0}^{\xi} d \zeta=\sqrt{3} \int_{v}^{\widetilde{v}} \frac{d \phi}{\left(\phi^{2}-\bar{v}^{2}\right) \sqrt{\tilde{v}^{2}-\phi^{2}}} .
$$

Then after some algebraic calculations we get solitary wave solutions:

$$
v(\xi)= \pm \frac{\bar{v} \widetilde{v}}{\sqrt{\left(\widetilde{v}^{2}-\bar{v}^{2}\right) \tanh ^{2}\left(\bar{v} \xi \sqrt{\left(\widetilde{v}^{2}-\bar{v}^{2}\right) / 3}\right)+\bar{v}^{2}}} .
$$

Along the above heteroclinic orbit we have $y=\left(\bar{v}^{2}-v^{2}\right)$ $\sqrt{\left(\widetilde{v}^{2}-v^{2}\right) / 3}$. As above, we can obtain kink and antikink wave solutions:

$$
v(\xi)= \pm \frac{\overline{v y} \tanh \left(\bar{v} \xi \sqrt{\left(\widetilde{v}^{2}-\bar{v}^{2}\right) / 3}\right)}{\sqrt{\widetilde{v}^{2}+\bar{v}^{2}\left(\tanh ^{2}\left(\bar{v} \xi \sqrt{\left(\widetilde{v}^{2}-\bar{v}^{2}\right) / 3}\right)-1\right)}} .
$$

Region $\{I I\}$. There is eye-figure loop of system (7), defined by $H(v, y)=H(\sqrt{-b / 2}, 0)=H( \pm \bar{v}, 0)$, connecting the cuspidal points $( \pm \bar{v}, 0)$. Therefore $y= \pm \sqrt{\left(\bar{v}^{2}-v^{2}\right)^{3} / 3}$. Along the above heteroclinic orbit we have $y=\left(\bar{v}^{2}-v^{2}\right) \sqrt{\left(\bar{v}^{2}-v^{2}\right) / 3}$, which have kink and antikink solutions:

$$
v(\xi)= \pm \frac{\xi \bar{v}^{3}}{\sqrt{3+\xi^{2} \bar{v}^{4}}} .
$$

Region $\{V\}$. There are two orbits homoclinic to origin for system (7) defined by $H(v, y)=0$ that are passing through points $( \pm \bar{v}, 0)$. Therefore $y= \pm|v| \sqrt{\left(\bar{v}^{2}-v^{2}\right)\left(v^{2}+k^{2}\right) / 3}$, where $\bar{v}=\sqrt{-3 b / 4+\sqrt{9 b^{2}-48 a} / 4}$ and $k^{2}=3 b / 4+$ $\sqrt{9 b^{2}-48 a} / 4$. The solitary wave solutions are

$$
v(\xi)= \pm \frac{\sqrt{2} \bar{v} k}{\sqrt{\left(k^{2}-\bar{v}^{2}\right)+\left(k^{2}+\bar{v}^{2}\right) \cosh (2 \bar{v} k \xi / \sqrt{3})}} .
$$

Region $\{V I I\}$. There are two nilpotent homoclinic orbits of system (7), defined by $H(v, y)=0$ connecting to degenerate saddle point $(0,0)$ and passing through points $( \pm \widetilde{v}, 0)$. Then we have $y= \pm \sqrt{v^{4}\left(\widetilde{v}^{2}-v^{2}\right)}= \pm v^{2} \sqrt{\left(\widetilde{v}^{2}-v^{2}\right) / 3}$ where $\widetilde{v}=$ $\sqrt{-3 b / 2}$. The solitary wave solutions are

$$
v(\xi)= \pm \frac{\sqrt{3} \widetilde{v}}{\sqrt{3+\widetilde{v}^{4} \xi^{2}}} .
$$


Case $2(\gamma=-1)$

Region $\{I\}$. There are two heteroclinic orbits of system (7), defined by $H(v, y)=H( \pm \bar{v}, 0)=H\left( \pm \sqrt{\left(b+\sqrt{\Delta^{-}}\right) / 2}, 0\right)$ connecting the two saddle points $( \pm \bar{v}, 0)$. Therefore $y= \pm\left(\bar{v}^{2}-\right.$ $\left.v^{2}\right) \sqrt{\left(v^{2}+k^{2}\right) / 3}$, where $k^{2}=-b / 2+\sqrt{\Delta^{-}}$, which have kink and antikink solutions:

$$
v(\xi)= \pm \frac{\bar{v} k \tanh \left(\bar{v} \xi \sqrt{\left(\bar{v}^{2}+k^{2}\right) / 3}\right)}{\sqrt{k^{2}+\bar{v}^{2}\left(1-\tanh \left(\bar{v} \xi \sqrt{\left(\bar{v}^{2}+k^{2}\right) / 3}\right)\right.}}
$$

Region $\{I I\}$. There are two heteroclinic orbits of system (7), defined by $H(v, y)=H( \pm \sqrt{b}, 0)$ connecting with tow saddle points $( \pm \sqrt{b}, 0)$. Therefore $y= \pm\left(b-v^{2}\right) \sqrt{\left(v^{2}+b / 2\right) / 3}$. The kink and antikink solutions are given by

$$
v(\xi)= \pm \frac{\sqrt{b} \tanh (b \xi / \sqrt{2})}{\sqrt{3-2 \tanh ^{2}(b \xi / \sqrt{2})}} .
$$

Region $\{I I I(a)\}$. There are two homoclinic orbits of system (7) defined by $H(v, y)=0$ connecting with saddle point $(0,0)$ and passing through points $( \pm \bar{v}, 0)$. Therefore

$$
y= \pm|v| \sqrt{\frac{\left(v^{2}-\bar{v}^{2}\right)\left(v^{2}-k^{2}\right)}{3}}
$$

where $\bar{v}=\sqrt{3 b-\sqrt{9 b^{2}+48 a}} / 2$ and $k=\sqrt{3 b+\sqrt{9 b^{2}+48 a}} / 2$, of which we have two solitary wave solutions:

$$
v(\xi)= \pm \frac{2 \bar{v} k \sqrt{k^{2}-\bar{v}^{2}} \exp (\xi \bar{v} k / \sqrt{3})}{\sqrt{\left[\left(k^{2}-\bar{v}^{2}\right) \exp (2 \xi \bar{v} k / \sqrt{3})\right]^{2}-4 \bar{v}^{2} k^{2}}} .
$$

In this region there are also two heteroclinic orbits of system (7), defined by $H(v, y)=H( \pm \widetilde{v}, 0)=H\left( \pm \sqrt{\left(b+\sqrt{\Delta_{-}}\right) / 2}, 0\right)$ connecting the saddle points $( \pm \widetilde{v}, 0)=\left( \pm \sqrt{\left(b+\sqrt{\Delta_{-}}\right) / 2}, 0\right)$. Therefore $y= \pm\left(\widetilde{v}^{2}-v^{2}\right) \sqrt{\left(v^{2}+m^{2}\right) / 3}$, where $k^{2}=(-b+$ $\left.2 \sqrt{\Delta_{-}}\right) / 2$. Kink and antikink solutions are given by

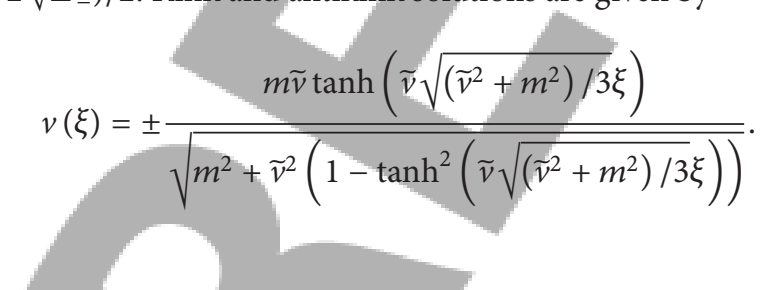

Region $\{I I I(b)\}$. There are four heteroclinic orbits of system (8) defined by $H(v, y)=H(0,0)$. Two of these orbits connect $(0,0)$ and $\left(-\sqrt{\left(b+\sqrt{\Delta_{-}}\right) / 2}, 0\right)$ and the other two connect $(0,0)$ and $\left(\sqrt{\left(b+\sqrt{\Delta_{-}}\right) / 2}, 0\right)$. Therefore $y=$ $\pm \sqrt{\left(\bar{v}^{2}-v^{2}\right)^{2} v^{2} / 3}= \pm\left(\bar{v}^{2}-v^{2}\right) v / \sqrt{3}$, where $\bar{v}=\sqrt{\left(b+\sqrt{\Delta_{-}}\right) / 2}$. Kink and antikink solutions are of the form

$$
v(\xi)= \pm \frac{\bar{v} \widetilde{v} \exp \left(\bar{v}^{2} \xi / \sqrt{3}\right)}{\sqrt{\bar{v}^{2}+\widetilde{v}^{2}\left(\exp \left(2 \bar{v}^{2} \xi / \sqrt{3}\right)-1\right)}},
$$

where $\widetilde{v}=\sqrt{\left(b-\sqrt{\Delta_{-}}\right) / 2}$.

Region $\{I I I(c)\}$. There are two homoclinic orbits of system (7) defined by $H(v, y)=H\left( \pm \sqrt{\left(b+\sqrt{\Delta_{-}}\right) / 2}, 0\right)=H( \pm \bar{v}, 0)$ connecting with saddle points $( \pm \bar{v}, 0)$ and passing through points $( \pm \widetilde{v}, 0)$. Therefore $y= \pm\left(\bar{v}^{2}-v^{2}\right) \sqrt{\left(v^{2}-\widetilde{v}^{2}\right) / 3}$, where $\bar{v}=\sqrt{\left(b+\sqrt{\Delta_{-}}\right) / 2}$ and $\tilde{v}=\sqrt{\left(b-2 \sqrt{\Delta_{-}}\right) / 2}$. There are solitary wave solutions which are given by

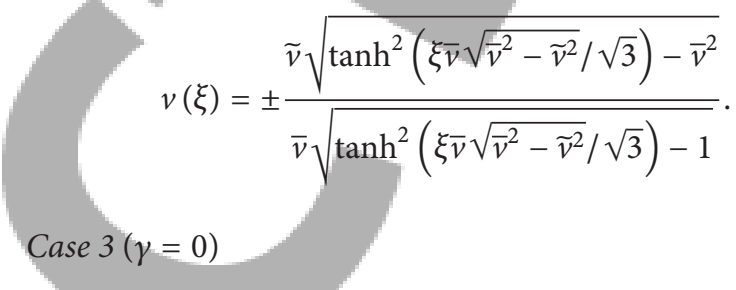

Region $\{b=1, a<0\}$. There are two orbits homoclinic to origin for system (7) defined by $H(v, y)=0$ which are passing through points $( \pm \sqrt{-2 a}, 0)$. Therefore $y=$ $\pm|v| \sqrt{-\left(v^{2}+2 a\right) / 2}$. Two solitary wave solutions are given by

$$
v(\xi)= \pm \sqrt{2 a \cot ^{2}\left(\sqrt{-a} \xi+\frac{\pi}{2}\right)-2 a} .
$$

Region $\{b=-1, a>0\}$. There are two heteroclinic orbits of system (7), defined by $H(v, y)=H( \pm \sqrt{a}, 0)$ connecting saddle points $( \pm \sqrt{a}, 0)$ defined by $y= \pm\left(a-v^{2}\right) / \sqrt{2}$. Kink and antikink solutions are

$$
v(\xi)= \pm \sqrt{a} \tan \left(\sqrt{\frac{a}{2}} \xi\right)
$$

Case 4 (periodic orbits). Now we calculate explicit formulas for bounded periodic travelling waves. Here we only consider periodic orbits of (7) which are located inside the right homoclinic loops of Figure $2(\gamma=-1)$ in regions III(a) and III(c) and inside the right heteroclinic loop of Figure $2(\gamma=$ $-1)$ in region III(b). Suppose that the periodic orbits pass through $\left(\omega_{1}, 0\right)$ and $\left(\omega_{2}, 0\right)$ so that $0<\omega_{1}<\omega_{2}$. Therefore this periodic orbit lies on level curve $H(v, y)=H\left(\omega_{2}, 0\right)=$ $h$ where $H$ is the Hamiltonian function. Define $G(v, 0)=$ $H\left(\omega_{2}, 0\right)-H(v, 0) . G(v, 0)$ is a sixth order polynomial with respect to $v$, where $\pm \omega_{1}, \pm \omega_{2}$, and $\pm \omega_{3}$ are their roots with $0<\omega_{1}<\omega_{2}<\omega_{3}$. Therefore

$$
y= \pm \sqrt{2 G(v, 0)}= \pm \sqrt{\frac{\left(v^{2}-\omega_{1}^{2}\right)\left(v^{2}-\omega_{2}^{2}\right)\left(v^{2}-\omega_{3}^{2}\right)}{3}} .
$$

Since $d v / d \xi=y$, we get

$$
\int_{0}^{\xi} d \zeta=\sqrt{3} \int_{\omega_{1}}^{v} \frac{d \phi}{\sqrt{\left(\phi^{2}-\omega_{1}^{2}\right)\left(\phi^{2}-\omega_{2}^{2}\right)\left(\phi^{2}-\omega_{3}^{2}\right)}} .
$$


With change of variable of the form $\phi^{2}=u$ on the right hand side above, we can derive

$$
\int_{0}^{\xi} d \zeta=\frac{\sqrt{3}}{2} \int_{\omega_{1}^{2}}^{v^{2}} \frac{d u}{\sqrt{u\left(u-\omega_{1}^{2}\right)\left(u-\omega_{2}^{2}\right)\left(u-\omega_{3}^{2}\right)}} .
$$

Now by using integral tables for elliptic integrals [21] we have

$$
\frac{2 \xi}{\sqrt{3}}=g \int_{0}^{u_{1}} d u=g u_{1}=g s n^{-1}(\sin \phi, k)
$$

where $s n^{-1}$ is inverse Jacobian elliptic function with modulus $k$ (see [21]), $s n u_{1}=\sin \varphi$, and

$$
\begin{gathered}
\phi=\sin ^{-1}\left(\sqrt{\frac{\omega_{2}^{2}\left(v^{2}-\omega_{1}^{2}\right)}{v^{2}\left(\omega_{2}^{2}-\omega_{2}^{1}\right)}}\right), \quad k^{2}=\frac{\left(\omega_{2}^{2}-\omega_{1}^{2}\right) \omega_{3}^{2}}{\left(\omega_{3}^{2}-\omega_{1}^{2}\right) \omega_{2}^{2}}, \\
g=\frac{2}{\sqrt{\left(\omega_{3}^{2}-\omega_{1}^{2}\right) \omega_{2}^{2}}} .
\end{gathered}
$$

Then after some algebraic calculations we get solitary wave solution:

$$
v(\xi)=\frac{\omega_{1}^{2} \omega_{2}^{2}}{\omega_{2}^{2}+\left(\omega_{1}^{2}-\omega_{2}^{2}\right) s n^{2}\left(\xi \sqrt{\left(\omega_{3}^{2}-\omega_{1}^{2}\right) \omega_{2}^{2}} / 2\right)} .
$$

Formulas for other periodic orbits of system (7) can be derived in a similar manner which we omitted here for the sake of brevity.

\section{Conflict of Interests}

The author declares that there is no conflict of interests regarding the publication of this paper.

\section{References}

[1] R. Hirota, "Exact solution of the korteweg_de vries equation for multiple Collisions of solitons," Physical Review Letters, vol. 27, no. 18, pp. 1192-1194, 1971.

[2] R. Hirota, The Direct Method in Soliton Theory, vol. 155 of Cambridge Tracts in Mathematics, Cambridge University Press, Cambridge, UK, 2004.

[3] M. J. Ablowitz and P. A. Clarkson, Soliton, Nonlinear Evolution Equations and Inverse Scattering, vol. 149 of London Mathematical Society Lecture Note Series, Cambridge University Press, Cambridge, Mass, USA, 1991.

[4] C. Rogers and W. K. Schief, Bäcklund and Darboux Transformations, Geometry and Modern Applications in Soliton Theory, Cambridge Texts in Applied Mathematics, Cambridge University Press, Cambridge, UK, 2002.

[5] P. J. Olver, Applications of Lie Groups to Differential Equations, Springer, New York, NY, USA, 1993.

[6] E. G. Fan, Integrable Systems and Computer Algebra, Science Press, 2004.

[7] C. Yan, "A simple transformation for nonlinear waves," Physics Letters A, vol. 224, no. 1-2, pp. 77-84, 1996.
[8] J. He, "Application of homotopy perturbation method to nonlinear wave equations," Chaos, Solitons and Fractals, vol. 26, no. 3, pp. 695-700, 2005.

[9] M. L. Wang, "Exact solutions for a compound KdV-Burgers equation," Physics Letters A: General, Atomic and Solid State Physics, vol. 213, no. 5-6, pp. 279-287, 1996.

[10] H. Junqi, "An algebraic method exactly solving two highdimensional nonlinear evolution equations," Chaos, Solitons and Fractals, vol. 23, no. 2, pp. 391-398, 2005.

[11] S. Liu, Z. Fu, and Q. Zhao, "Jacobi elliptic function expansion method and periodic wave solutions of nonlinear wave equations," Physics Letters A, vol. 285, no. 1-2, pp. 69-74, 2001.

[12] J. Li and L. Zhang, "Bifurcations of traveling wave solutions in generalized Pochhammer-Chree equation," Chaos, Solitons and Fractals, vol. 14, no. 4, pp. 581-593, 2002.

[13] J. Li and T. L. He, "Exact traveling wave solutions and bifurcations in a nonlinear elastic rod equation," Acta Mathematicae Applicatae Sinica, vol. 26, no. 2, pp. 283-306, 2010.

[14] V. E. Zakharov and A. B. Shabat, "Exact theory of twodimensional self-focusing and one dimensionsl self-modulation of waves in nonlinear media," Journal of Experimental and Theoretical Physics, vol. 34, no. 1, pp. 62-69, 1972.

[15] E. Kit and L. Shemer, "Spatial versions of the Zakharov and Dysthe evolution equations for deep-water gravity waves," Journal of Fluid Mechanics, vol. 450, pp. 201-205, 2002.

[16] M. V. Goldman, "Strong turbulence of plasma waves," Reviews of Modern Physics, vol. 56, no. 4, pp. 709-735, 1984.

[17] D. Anderson, "Variational approach to nonlinear pulse propagation in optical fibers," Physical Review A, vol. 27, no. 6, pp. 3135-3145, 1983.

[18] V. E. Zakharov, “Collapse of Langmuir waves," Soviet Physics JETP, vol. 35, no. 5, pp. 908-914, 1972.

[19] V.E. Zakharov and V.S. Synakh, "The nature of the self-focusing singularity," Soviet Physics-JETP, vol. 41, pp. 465-468, 1976.

[20] J. K. Hale and H. Koçak, Dynamics and Bifurcation, Springer, New York, NY, USA, 1991.

[21] P. F. Byrd and M. D. Friedman, Handbook of Elliptic Integrals for Engineers and Scientists, Springer, Berlin, Germany, 1971. 